\title{
Kanon und Konflikt. Kunst aus dem Nationalsozialismus in deutschen Kunstmuseen
}

\section{Darja Jesse}

Technische Universität Berlin

\section{Abstract}

Nur wenige Kunstmuseen in Deutschland zeigen in ihren Dauerausstellungen Kunst, die während des Nationalsozialismus regimekonform entstand. In diesem Aufsatz untersuche ich die Präsentation dieser Kunst im Germanischen Nationalmuseum in Nürnberg, im Städel Museum in Frankfurt am Main und in der Pinakothek der Moderne in München. Über den Begriff des Kanons nähere ich mich den Ausstellungsnarrativen, in welche diese Kunst eingebettet ist.

Seuls quelques musées d'art en Allemagne montrent les œuvres d'art créées et présentées durant le national-socialisme dans leurs expositions permanentes. Cet article examine la présentation de ces œuvres d'art au Germanisches Nationalmuseum de Nuremberg, au Städel Museum de Francfort-sur-le-Main et à la Pinakothek der Moderne de Munich. En partant du concept de canon artistique, j'analyse les récits d'exposition dans lesquels cet art s'inscrit.

Only a few art museums in Germany show in their permanent exhibitions art that was created and officially presented during National-Socialism. This paper examines the presentation of such artworks in the Germanisches Nationalmuseum in Nuremberg, the Städel Museum in Frankfurt am Main, and the Pinakothek der Moderne in Munich. Through the concept of the canon, I approach exhibition narratives that frame the presentation of these artworks.

\section{Keywords}

Museum • Ausstellung • Nationalsozialismus • Schwieriges Erbe

Museum • Exhibition • National-Socialism • Difficult Heritage

Musée $\cdot$ Exposition $•$ National-Socialisme $\cdot$ Héritage difficile

\section{EINLEITUNG}

Museen sind nicht nur diejenigen Institutionen, die kulturelles Erbe sammeln, bewahren, erforschen, ausstellen und vermitteln - wie es in der derzeitigen Museumsdefinition des Deutschen Museumbunds heißt (Deutscher Museumsbund 2006: 6). Sie sind, in der Quintessenz dieser Tätigkeiten, vor allem maßgeblich daran beteiligt, das kulturelle Erbe überhaupt erst zu selektieren und zu konstituieren. Welche Objekte in die Sammlung gelangen und welche Sammlungsobjekte in der Dauerausstellung gezeigt werden, ist das Ergebnis von Entscheidungsprozessen, die von den historischen, gesellschaftlichen und kulturpolitischen Rahmenbedingungen, aber auch von institutionellen und individuellen Positionierungen bestimmt sind.
Kunstmuseen präsentieren ihre Sammlungen zumeist chronologisch nach kunsthistorischen Kategorien - aufgeteilt nach Kunstströmungen, Schulen odergeografischen Räumen. Die Werke sind ferner nach Format, Gattung, Material, Sujet oder Künstler*in zusammengestellt. Roswitha Muttenthaler und Regina Wonisch haben in ihrem Buch Gesten des Zeigens auf den Ursprung solcher Ausstellungsdisplays hingewiesen: „Die Anordnung der Gemälde, die so selbstverständlich und unhinterfragt die Präsentationsweise in kunsthistorischen Museen prägt, dass sie schon beinahe natürlich wirkt, ist Ausdruck des spezifischen Wissenschaftsverständnisses des 19. Jahrhunderts" (Muttenthaler/Wonisch 2006: 123f.). Diese Art von Erzählung etablierte sich zudem mit dem im 20. 
Jahrhundert immer stärker werdenden Selbstverständnis der Kunstmuseen, als Bildungseinrichtungen für die Vermittlung der Kunstgeschichte an eine breite Öffentlichkeit verantwortlich zu sein. Vergleicht man die Präsentation der Kunst aus der ersten Hälfte des 20. Jahrhunderts in größeren Kunstmuseen, die auf eine lange Institutionsgeschichte zurückblicken können und über umfangreiche Sammlungen verfügen, werden dem Publikum, zumeist der Reihe nach, Räume zu Impressionismus, Expressionismus, Kubismus, Bauhaus, Neuer Sachlichkeit und Nachkriegsmoderne dargeboten. Die Sammlungsschwerpunkte der Museen variieren, aber sie sind zugleich einem kunsthistorischen Kanon verpflichtet.

Obgleich der Begriff „Kanon“ im kunsthistorischen Kontext selbstverständlich erscheint - ob normativ oder kritisch verstanden -, ist die Forschungsliteratur dazu, anders als im Bereich der Literaturwissenschaften, überschaubar. ${ }^{1}$ Ich beziehe mich im Folgenden auf die vom Kunsthistoriker Hubert Locher vorgeschlagene Definition des Kanons „als Bezeichnung für eine Gruppe von Werken der Kunst [...], die innerhalb einer bestimmten sozialen Gemeinschaft als exemplarisch und für bestimmte Wertvorstellungen stehend betrachtet und erinnert werden. [...] Entscheidend ist, dass in dieser Konzeption die narrativ konstruierte Reihe als vorgefundene, natürliche Ordnung aufgefasst wird. Als Akteure dieser Geschichte der Kunst werden die ,Meister' und ihre Produkte, die ,Meisterwerke' aufgefasst“ (Locher 2013: 364). Die Kunst aus dem Nationalsozialismus findet sich allerdings selten in diesem Narrativ wieder. Auf die Kunst aus der Weimarer Republik folgt in den Dauerausstellungen der Kunstmuseen meist die Nachkriegszeit. „Bis heute, bis 2015, zeigt kein deutsches Kunstmuseum in seiner ständigen Sammlung bzw. in seiner Dauerausstellung Beispiele der nationalsozialistischen Kunst", konstatierte Christian Fuhrmeister (2015a: 97f.) noch vor wenigen Jahren. In den vergangenen sechs Jahren gab es in dieser Hinsicht jedoch Veränderungen und Werke, die unter dem Nationalsozialismus entstanden, wurden in die Dauerausstellungen von Kunstmuseen integriert. Im Städel Museum in Frankfurt am Main und in der Pinakothek der Moderne in München wurden eigene Säle zur Kunstproduktion im Nationalsozialismus eingerichtet, welche ich im Folgenden genauer vorstellen werde. Da ihrer Konzeption und Rezeption ein jahrzehntelanger Disput zum Umgang mit dem nationalsozialistischen Erbe vorangegangen war, werde ich diesen schlaglichtartig beleuchten. Vor diesem Hintergrund beziehe ich auch ein drittes Museum in die Betrachtung ein - das Germanische Nationalmuseum in Nürnberg, dessen Saal zur Kunstproduktion im Nationalsozialismus in den 1990er-Jahren eingerichtet wurde.

\section{„NAZI-KUNST INS MUSEUM?“}

Lange hat sich die Kunstgeschichte mit der Kunst, die zwischen 1933 und 1945 entstand und mit der offiziellen

\footnotetext{
1 Einen guten Forschungsüberblick zum Terminus „Kanon“ bietet Hoffmann (2013 136-140). Überdies sei insbesondere auch auf Locher (2013) und Langfeld (2018) hingewiesen.
}

Kunstpolitik des NS-Regimes einherging, nicht beschäftigt. Sie wurde als Propaganda, Kitsch oder „Unkunst" ${ }^{2}$ abgewertet und damit außerhalb des Gegenstandsbereichs der Kunstgeschichte verortet. Dass die Künstler, die sie produzierten, meist vor 1933 und eine Reihe von innen auch nach 1945 künstlerisch tätig waren, wurde nicht thematisiert (Brauneis 2020; Brauneis/Gross 2021). Die Beschäftigung mit der Kunst aus dem Nationalsozialismus fand zudem weitgehend abgekoppelt von der Erforschung der ästhetischen Moderne statt und vice versa.

Dass die Dichotomie - die guten Künste der Moderne auf der einen, die bösen Künste des NS-Regimes auf der anderen Seite - nicht weiter haltbar ist, wurde in den letzten Jahren in mehreren Sonderausstellungen aufgezeigt. Im Frühjahr 2019 eröffneten in Berlin die Schau „Emil Nolde - eine deutsche Legende. Der Künstler im Nationalsozialismus“ im Hamburger Bahnhof - Museum für Gegenwart und die Ausstellung „Flucht in die Bilder? Die Künstler der Brücke im Nationalsozialismus" im Brücke-Museum. Die Ausstellungen und ihre umfassenden Begleitpublikationen rückten in den Fokus der Öffentlichkeit, dass auch Künstler der Moderne - hier des Expressionismus - von der antisemitischen Ideologie überzeugt sein konnten, wie etwa Emil Nolde (Soika/Fulda/ Ring 2019), oder sich mit dem neuen Regime zumindest zu arrangieren versuchten, wie Max Pechstein (Soika/Hoffmann 2019: 118ff.). Es galt, die Handlungsspielräume der Künstler während des Nationalsozialismus differenziert zu analysieren und das "Verfemungs- und Verfolgungsnarrativ“ (ebd.: 14), welches die ästhetische Moderne nach 1945 pauschal mit NS-Widerstand gleichsetzte, als ein Konstrukt der Nachkriegszeit aufzuzeigen. Tatsächlich bedeutete die Einstufung von Kunstwerken als "entartet“ nicht automatisch, dass deren Produzenten die rassistisch-antisemitische Ideologie ablehnten. ${ }^{3}$

Diese beiden Ausstellungen repräsentieren gewissermaßen den heutigen Status quo im Umgang mit der Kunst, die zum kunsthistorischen Kanon gehört, aber im Hinblick auf die Weltanschauung ihrer Produzenten im Nationalsozialismus einer Kontextualisierung bedarf. Ihnen gingen jedoch jahrzehntelange Debatten voraus. Die zentrale Frage lautete: Darf die Kunst aus dem Nationalsozialismus in musealen Räumen präsentiert werden und wenn ja wie? Im Fokus stand allerdings lange nur diejenige Kunst, die in der Nachkriegszeit jenseits des Kanons verortet wurde und seitdem als problematisch galt - die Kunst, die regimekonform produziert und präsentiert wurde.

Die erste Ausstellung zur Kunst aus dem Nationalsozialismus wurde 1974 vom Frankfurter Kunstverein unter dem Titel Kunst im 3. Reich. Dokumente der Unterwerfung ausgerichtet. Es sei hervorgehoben, dass sie von einem Kunstverein und nicht von einem Museum initiiert wurde. Nachdem die Schau in Frankfurt am Main zu sehen war, 2 Den Begriff der „Unkunst“ prägte Georg Imdahl (1988).
3 Mit diesem Themenkomplex befasste sich im Mai 2019 auch das Kolloquium „Un-
bewältigt? Ästhetische Moderne und Nationalsozialismus. Kunst, Kunsthandel, Ausstel-
lungspraxis“, dessen Vorträge im gleichnamigen Sammelband erschienen sind (siehe Hoffmann/Scholz 2020). 
wurde sie im darauffolgenden Jahr in Hamburg, Stuttgart, Ludwigshafen, Wuppertal und Berlin gezeigt. Die Exponate - bei denen es sich in vielen Fällen nur um fotografische Reproduktionen handelte - stammen aus den Bereichen Architektur, Plastik und Malerei. Die von den Kunsthistorikern Georg Bussmann und Berthold Hinz kuratierte Schau sollte „nicht als reine Kunstausstellung angesehen werden, sondern eher als eine Dokumentation, die zeigt, in welchem Maße die Kunst welcher Politik diente" (Bussmann 1975: 3). Ausgewählt wurden solche Werke, die zwischen 1937 und 1944 in den „Großen Deutschen Kunstausstellungen“ im Haus der Deutschen Kunst in München präsentiert worden waren oder in der offiziellen Publizistik des Regimes, wie den Zeitschriften „Die Kunst im Deutschen Reich“ und „Die Baukunst", erschienen waren (ebd.)

Zur Frankfurter Ausstellung erschien nicht nur ein begleitender Katalog, sondern ein weiterer, nach der Laufzeit der Schau veröffentlichter Band mit dem Titel Betrifft: Reaktionen (Frankfurter Kunstverein 1975). Dieser enthält Fotos der Ausstellung und Ausstellungstexte, das Feedback der Besucher*innen auf einer Wandzeitung, die Auswertung einer vom Frankfurter Kunstverein im Vorfeld der Schau in Auftrag gegebene Besucher*innenumfrage, den Pressespiegel und die als Proteste gegen die Ausstellung veröffentlichten Flugblätter und Erklärungen (u.a. vom Verband Bildender Künstler). Die Komplexität des Ausstellungsvorhabens und die zu erwartende Kritik waren den Machern bewusst, daher hatten sie auf ausführliche Texttafeln sowie eine Feedbackwand gesetzt und eine Besucher*innenumfrage initiiert. Sie offerierten dem Publikum damit ein umfassendes Kommunikations- und Dialogangebot.

Nach dieser Schau folgten bis heute zwar etliche weitere Ausstellungen zur Kunst aus dem Nationalsozialismus, doch Kunst im 3. Reich sollte in mehrfacher Hinsicht prägend wirken: Zum einen bestimmte die Schau durch ihre Objektauswahl nachhaltig das Bild dessen, was als „NSKunst" verstanden und diskutiert werden sollte; zum anderen kristallisierten sich im Zusammenhang mit dieser Ausstellung die Argumentationsmuster heraus, die bis in die 1990er anhalten sollten. Zu den letzteren zählte die Idee, mit Hilfe von „Schrifttafeln gegen die Aura von [...] Großgemälden an[zu]kämpfen“ (ebd.: 155), wie es in einem Presseartikel des Spiegel hieß. Dies sollte gewährleisten, dass das Publikum die Exponate als „Dokumente der Unterwerfung“ und nicht des ästhetischen Genusses wegen betrachten würde. „Allzuleicht könnten die Malwerke“, so der Autor, „wenn schon nicht kennerischen Enthusiasmus, so doch unbeschwertes Amüsement auslösen“.

Auf dieser Grundlage wurde in Deutschland in den 1980er-Jahren eine hitzige Debatte geführt, ob die Kunst aus dem Nationalsozialismus öffentlich gezeigt werden sollte oder nicht. Zum „Kulminationspunkt in der Rezeptionsgeschichte der NS-Kunst" (Berswordt-Wallrabe 2016: 11) wurde Klaus Staecks Veröffentlichung Nazi-Kunst ins Museum? (Staeck 1988). Der Auslöser war die avisierte Aufstellung der Porträtbüsten des Kunstsammlers Peter Ludwig und dessen Ehefrau Irene. Der Künstler: Arno Breker, einer der prominentesten
Bildhauer im Nationalsozialismus. Klaus Staeck, ein studierter Jurist und zu diesem Zeitpunkt bereits ein international renommierter Künstler, scheute sich nicht, politische Themen mit bissiger Satire zu kommentieren. Seinem Buch ging der Aufruf „Keine Nazi-Kunst in unsere Museen“ voraus, der von zahlreichen Künstler*innen, Kunsthistoriker*innen und Museumsmitarbeiter*innen unterzeichnet wurde (ebd.: 19). Die Argumentation setzte eine scharfe Trennung zwischen Verfolgten und Nutznießern des Regimes voraus: „Die großen Künstler unseres Jahrhunderts wurden verfolgt, mit Berufsverbot belegt, ermordet und ins Exil getrieben" (ebd.), ist in dem Aufruf zu lesen. Und noch schwerwiegender wogen die Argumente zu Qualität und Moral:

Nazi-Kunst von künstlerischer Qualität ist uns nicht bekannt [...]. Die deutschen Museen werden deshalb das fortsetzen, was sie in den letzten 40 Jahren in ihrem Wirken für die Kunst getan haben: sie werden Maßstäbe setzen. Kunst hat auch mit Ethik zu tun. Deshalb rufen wir erneut öffentlich auf: Nazi-Kunst gehört nicht in unsere Museen (ebd.)

Die Haltung, von der diese Argumentation getragen wurde, bringt Sonja Weishaupt kritisch auf dem Punkt: „Dieser Vorstellung von einem beschützen- und bewahrenswerten (und offenbar objektiv evidenten), Kanon ,wahrer' Kunst stünden die Werke von 1933-45 entgegen, nicht einmal gegenüber, da sie als ,Un-Kunst' mit ersterem nicht in Bezug gebracht werden können" (Weishaupt 2005: 32). Dass diese Kategorien die Kunstproduktion während des Nationalsozialismus nicht hinreichend erfassen, wurde nicht zuletzt in den erwähnten Ausstellungen zu Emil Nolde und den Brücke-Künstlern 2019 evident: Kunst, die als qualitativ hochrangig gilt, kann auch von einem mit dem Nationalsozialismus sympathisierenden Künstler geschaffen worden sein, wodurch die Kategorie „Ethik“ jeglicher Grundlage beraubt wird. Die Kunsthistorikerin Marlies Schmidt bemerkt in ihrer Forschung zur Kunst im Nationalsozialismus zudem treffend: Als über das Thema „NS-Kunst ins Museum“ gestritten wurde, gab es diese Kunst bereits längst in musealen Sammlungen (Schmidt 2012: 130). Teils, weil die Museen während des Nationalsozialismus Werke zeitgenössischer Künstler gekauft, teils, weil sie nach Kriegsende umfassende Konvolute zur Aufbewahrung bekommen hatten. Die Kunst aus dem Nationalsozialismus war in den Museen vorhanden, nur ausgestellt wurde sie lange Zeit nicht.

\section{KUNST AUS DEM NATIONALSOZIALISMUS IN DAUERAUSSTELLUNGEN}

Die Dauerausstellung ist die „Visitenkarte“ eines Museums, denn in ihr werden „repräsentative Objekte einer musealen Sammlung dauerhaft [...] einem größtmöglichen Publikum zugänglich gemacht" (Habsburg-Lothringen 2012: 9). Während Sonderausstellungen einzelne Fragestellungen sowie Kontexte - und in Kunstmuseen auch einzelne Künstler*innen und Kunstströmungen - fokussieren, sind 
dauerhafte Präsentationen häufig durch einen Überblickscharakter gekennzeichnet.

Bis vor wenigen Jahren war Kunst aus dem Nationalsozialismus ausschließlich in den Dauerausstellungen von historischen und kulturhistorischen Museen zu sehen. In einem (kultur-)historischen Ausstellungsdisplay werden Exponate wie Gemälde, Zeichnungen oder Plastiken aus dem Nationalsozialismus jedoch weniger als autonome Kunstwerke präsentiert, sondern vielmehr als Beispiele für die Bildpropaganda in einen breit gefassten Kontext der nationalsozialistischen (Kultur-)Politik eingebettet. Meistens werden Kunstwerke und andere Objektgattungen - wie Objekte der Alltagskultur, Militaria, Schriftstücke oder Filmaufnahmen gleichberechtigt in eine Erzählung einbezogen und miteinander verschränkt. Anders verhält es sich mit Kunstwerken aus dem Nationalsozialismus in einem Kunstmuseum, dessen Exponate hauptsächlich nach formalästhetischen Kategorien vermittelt und rezipiert werden.

In ihren Überblickskatalogen und auf den Websites betonen die drei hier betrachteten Museen die Qualität und Bandbreite ihrer Sammlungen. Auf der Website des Städel Museums ist zu lesen: „Unter einem Dach bietet die Sammlung des Städel Museums einen nahezu lückenlosen Überblick über 700 Jahre europäische Kunstgeschichte."4 Das Germanische Nationalmuseum biete im Ausstellungsbereich zum 20. Jahrhundert ein „umfassendes Bild der Kunst- und Stilgeschichte "5 und zur Pinakothek der Moderne heißt es, dass sie „innerhalb weniger Jahrzehnte quantitativ und qualitativ Weltniveau erreichte". ${ }^{6}$ Für Kunst aus dem Nationalsozialismus - die üblicherweise nicht unter den Kategorien „Weltrang“, „Meisterwerk" und „Qualität" subsumiert wird - scheint es in diesem Narrativ zunächst keinen Platz zu geben. Aber die Institution Museum wandelt sich - auch in Wechselwirkung mit den Dynamiken in der Fachdisziplin Kunstgeschichte -, neue Narrative ergänzen oder ersetzen die bekannten und an die bestehenden Sammlungen werden neue Fragen gerichtet. Das Medium der Dauerausstellung ist weitaus dynamischer als seine Bezeichnung zunächst vermuten lässt.

\section{Germanisches Nationalmuseum, Nürnberg}

Das Germanische Nationalmuseum in Nürnberg ist das größte kulturhistorische Museum Deutschlands. Der 1997 kuratierte Ausstellungsbereich zum 20. Jahrhundert hat es zum Ziel, wie bereits zitiert, „ein umfassendes Bild der Kunstund Stilgeschichte der letzten 100 Jahre"7 zu präsentieren. Dieses Bild, so heißt es auf der Website zudem, „reicht von Meisterwerken der Malerei über ausdrucksstarke Skulpturen [...] bis hin zu [...] Klassikern der Designgeschichte“. Die zumeist als White Cube gestalteten Ausstellungsräume und

\footnotetext{
4 https://www.staedelmuseum.de/de/das-staedel-museum (Zugriff am 30. Novembe 2021).

5 https://www.gnm.de/ausstellungen/dauerausstellung/20-jahrhundert (Zugriff am 30. November 2021).

6 https://www.pinakothek.de/sammlung/sammlung-moderne-kunst (Zugriff am 30. November 2021).

7 https://www.gnm.de/ausstellungen/dauerausstellung/20-jahrhundert (Zugriff am 30. November 2021).
}

die weitestgehende Fokussierung auf die Präsentation von bildender Kunst, die nicht durch andere Objektgattungen kulturhistorisch ergänzt ist, ähneln vergleichbaren Sammlungspräsentationen in Kunstmuseen.

In diesen chronologischen, kunsthistorischen Erzählfaden ist der Saal zum Thema „Kunst im Nationalsozialismus“ eingeflochten (Abb. 1-3). Darin werden, wie das Publikum aus dem einführenden Wandtext erfährt, Werke aus den „Großen Deutschen Kunstausstellungen" gezeigt. Diese offiziellen Leistungsschauen der zeitgenössischen Kunst fanden zwischen 1937 und 1944 im eigens für sie errichteten Haus der Deutschen Kunst in München statt. Im Germanischen Nationalmuseum sind hauptsächlich Motive des Bauern- und Landlebens sowie Frauendarstellungen zu sehen: Mädchen am Weizenfeld von Elvira Bauer (1935), Erntepause von Georg

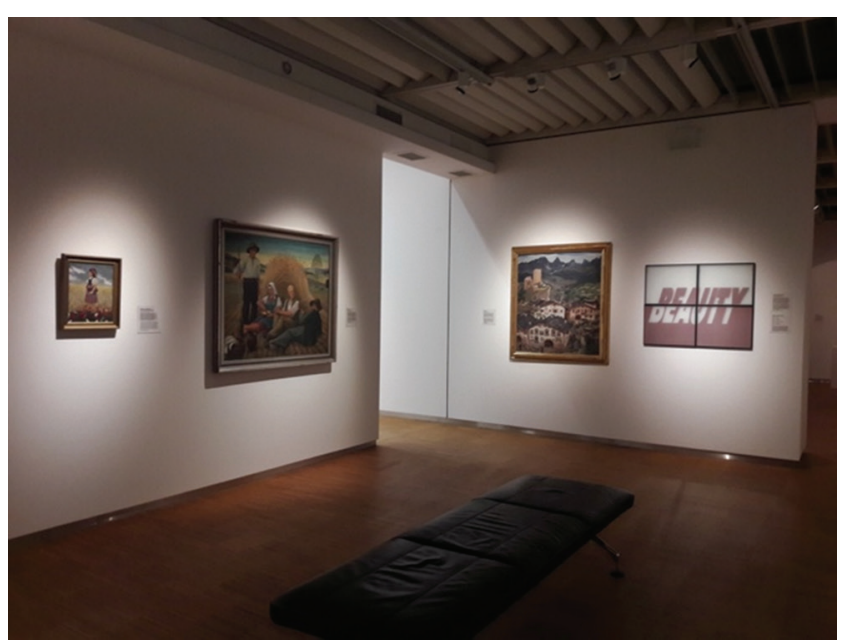

Abb. 1: Blick in den Saal „Kunst im Nationalsozialismus“ im Germanischen Nationalmuseum, Nürnberg. Linke Wand: Mädchen am Weizenfeld, Elvira Bauer (1935); Erntepause, Georg Günther (1938). Rechte Wand: Markt in Tirol, Erwin Puchinger (1939); Tafel Beauty. Look back von Jochen Gerz.

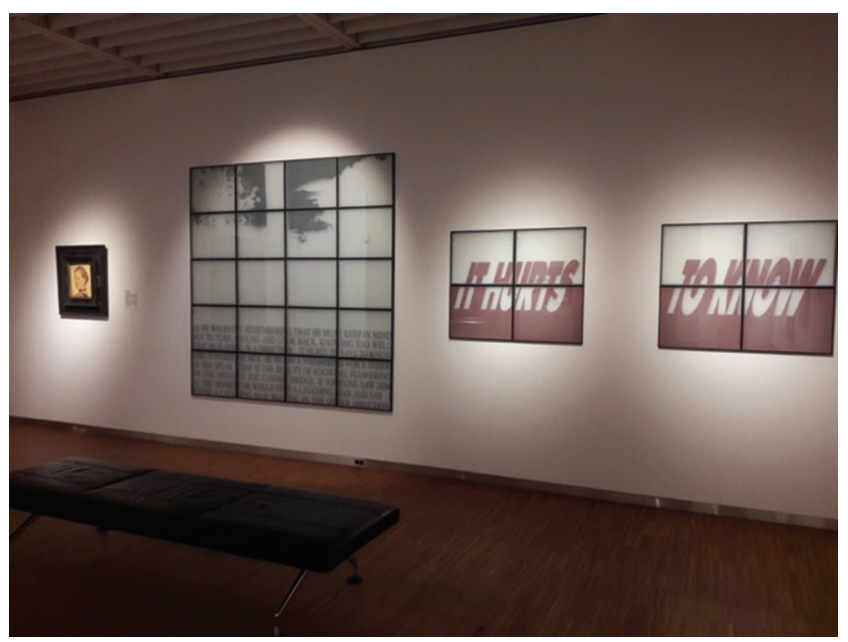

Abb. 2: Blick in den Saal "Kunst im Nationalsozialismus" im Germanischen Nationalmuseum, Nürnberg. V.I.n.r.: Die rote Halskette von Sepp Hilz (1942); Haupttafel sowie die Tafeln It hurts. To know this und To know. The distance von Jochen Gerz. 


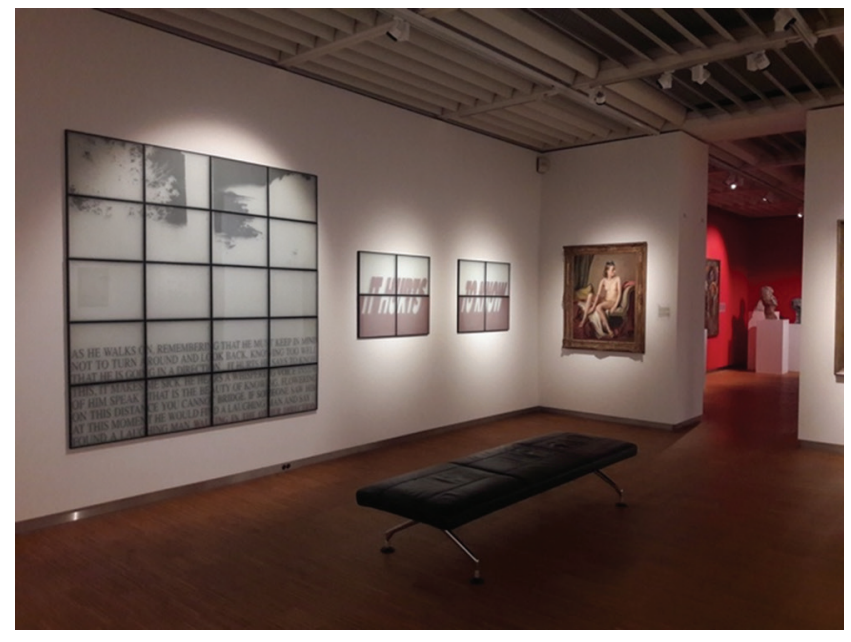

Abb. 3: Blick in den Saal „Kunst im Nationalsozialismus“ im Germanischen Nationalmuseum, Nürnberg. V.I.n.r.: Tafeln von Jochen Gerz, wie Abbildung 2; Akt, Adolf Ziegler (1942).

Günther (1938), Markt in Tirol von Erwin Puchinger (1939), Die rote Halskette von Sepp Hilz (1942), Akt von Adolf Ziegler (1942) sowie Um Haus und Hof von Adolf Reich (1940). ${ }^{8}$ In dem Einführungstext heißt es über die präsentierten Werke:

Sie huldigen dem Heimatidyll und dem „arischen“ Frauentypus, während Juden mit antisemitischen Klischees diffamiert werden. Diese „Völkischen“ Ideale sollten die Gesellschaft erziehen. Zeitgleich wurde die internationale Moderne als "entartet“ aus den Museen konfisziert, die Künstler wurden verfemt.

Die Objekttexte neben den einzelnen Exponaten setzen sich mit einigen der leitenden Ideen des Nationalsozialismus mit Blick auf das jeweilige Werk auseinander: Rasseideologie und Antisemitismus, „Blut-und-Boden“-Ideologie oder - wie bei den beiden im Nationalsozialismus populären Frauendarstellungen von Sepp Hilz und Adolf Ziegler - der Rolle der Frau als „Behüterin der Rasse“.

Was aus den erläuternden Wandtexten nicht hervorgeht, ist die Tatsache, dass alle hier gezeigten Werke - bis auf das Bild von Elvira Bauer, das auf keiner der „Großen Deutschen Kunstausstellungen“ ausgestellt worden war - von Adolf Hitler angekauft wurden. ${ }^{9}$ Die Werke stellen somit keinen Querschnitt der Kunst im Nationalsozialismus insgesamt dar, sondern sind eine sehr konzise und ausschließlich auf einen Akteur - Adolf Hitler als Kunstmäzen - fokussierte Auswahl. ${ }^{10}$ Durchbrochen ist diese Hängung durch die Arbeit Walter at the Helm of Beauty. Keeping in Mind (zu Deutsch: Walter am

8 Bis auf das Gemälde von Elvira Bauer, welches 2010 als Geschenk aus Privatbesitz 8 Bis auf das Gemälde von Elvira Bauer, welches 2010 als Geschenk aus Privatbesitz ausgestellten Werke Leihgaben. Sie stammen aus dem Konvolut "Ehemaliger Reichsbesitz", welches seit 1999 im Deutschen Historischen Museum verwahrt wird. Diese Provenienz ist auf den Objektschildern ausgewiesen.

9 Zu den ausgestellten Werken der "Großen Deutschen Kunstausstellungen“ siehe die Online-Datenbank GDK-Research unter http://www.gdk-research.de. Die Gemälde Erntepause von Georg Günther und Markt in Tirol wurden von Adolf Hitler 1939 für 2.500 RM und pause von Georg Günther und Markt in Tirol wurden von Adolf Hitler 1939 für 2.500 RM und .00 RM erworben, Um Haus und Hof von Adolf Reich kaufte er 1940 für 6.000 RM; im Ziegler (für 10.000 RM). 10 Ich danke Tilo Grabach, Leiter der Sammlung Kunst und Kunsthandwerk 19. bis 21 Jahrhundert, für den freundlichen Hinweis, dass Elvira Bauers Gemälde nicht mehr ausgestellt ist und dass an dessen Stelle seit November 2020 das Werk Verspottung (1933) von Josef Scharl, einem Maler der Neuen Sachlichkeit, zu sehen ist.
Steuer der Schönheit. Im Gedächtnis bewahren) von Jochen Gerz aus dem Jahr 1993. Das Werk besteht aus vier mit Texten und einer Fotografie bedruckten Glastafeln, welche zwischen den Gemälden aus den "Großen Deutschen Kunstausstellungen" platziert sind. Auf den drei kleineren Tafeln ist folgendes zu lesen: „Beauty. Look back“, „It hurts. To know this“ und „To know. The distance”. Dabei ziehen vor allem die groß gedruckten Begriffe „Beauty“ -,,It hurts“ -, ,To know" die Aufmerksamkeit auf sich und durch die räumliche Nähe zu den Frauendarstellungen von Hilz und Ziegler wirken sie als deren Kommentierung. Die größere Tafel ist mit dem Fotonegativ einer Landschaftsaufnahme und einem längeren Text bedruckt:

As he walks on, remembering that he must keep in mind not to turn around and look back, knowing too well that he is going in a direction... It hurts he says to know this, it makes me sick. He hears a whispering voice inside of him speak: That is the beauty of knowing, flowering on this distance you cannot bridge. If someone saw him at this moment he would find a laughing man and say: I found a laughing man walking in the other direction.

Jochen Gerz setzt sich in dieser Arbeit mit dem Nationalsozialismus auseinander, wie er selbst dem Germanischen Nationalmuseum mitteilte. Begriffe, welche unmittelbar darauf verweisen könnten, kommen auf den Tafeln jedoch nicht vor. Der Künstler nähert sich der Vergangenheit vielmehr assoziativ und poetisch über den Begriff der Schönheit und setzt sich auf diese Weise mit den Handlungsspielräumen von Kunst auseinander, aber auch mit der Rolle Nürnbergs und den dort aufwendig inszenierten Reichsparteitagen (Peters 2001: 8). In dem Objekttext zu Gerz' Werk ist die Interpretation derweil unmissverständlich formuliert:

Gerz' Arbeit fordert zum Nachdenken über die Instrumentalisierung der „Schönheit“ als Werbeträger „völkischer" Ideale in der Kunst des Dritten Reichs auf. Gegenüber der Moderne, die von den Nationalsozialisten als "entartet“ diffamiert und verfolgt wurde, griff die NS-Kunst auf vertraute und entsprechend leicht eingängige Vorbilder zurück. [...] Die Arbeit von Gerz berührt die Schnittstelle zwischen Vollkommenheitswunsch und Unmenschlichkeit.

Interventionen zeitgenössischer Künstler*innen sind ein verbreitetes Instrument, wenn es um die Präsentation von „schwierigem Erbe" geht. Der künstlerischen Arbeit von Jochen Gerz kommt im Germanischen Nationalmuseum eine vergleichbare Rolle zu. Auch die Chronologie der Dauerausstellung ist durchbrochen, um auf diese Weise den Werken aus den "Großen Deutschen Kunstausstellungen“ einen künstlerischen Kommentar der Gegenwart, also der späten 1990er-Jahre, entgegenzusetzen. Zwangsläufig drängt sich die Frage auf: Würde das Publikum die in diesem Saal gezeigte Kunst sonst missverstehen? Die Bilder einfach schön finden? Oder wie es Der Spiegel im erwähnten Artikel von 1974 beschrieb - „kennerischen Enthusiasmus“ oder „unbeschwertes Amüsement“ an den Tag legen? Angesichts der dargestellten Debatten der 1980er-Jahre, die sich weit in 
die 1990er fortsetzten, ist anzunehmen, dass die Konzeption des Saals auf die Kontroversen reagierte und einer eindeutigen Lektüre der präsentierten Werke klaren Vorzug gab. Das kuratorische Konzept spiegelt damit auch den zeitgenössischen Forschungsstand und die Tendenzen im Umgang mit der Kunst aus dem Nationalsozialismus wider: Vereindeutigung statt Ambivalenz. ${ }^{11}$

\section{Städel Museum, Frankfurt am Main}

Wie im Germanischen Nationalmuseum ist der Rundgang des Städel Museums chronologisch konzipiert. Von Dezember 2018 bis November 2019 wurde in einem Saal Kunst aus dem Nationalsozialismus ausgestellt. Der Raum trug den Titel "Instrumentalisierung von Kunst“..$^{12}$ Prominent durch deren zentrale Platzierung und starke Ausleuchtung war hier die Plastik Zehnkämpfer von Richard Scheibe aus dem Jahr 1936 präsentiert (Abb. 4). Über Richard Scheibe und seine Bronzeplastik erfuhr das Publikum nichts weiter, aber die rechts und links davon gezeigten Gemälde wurden im einführenden Wandtext kontextualisiert.

An der rechten Wand waren die Gemälde Der Krieg von Hans Thoma (1907) und Zwei nackte Männer mit Pferden von Fritz Boehle (ca. 1906) zu sehen (Abb. 5). Mag die Hängung dieser Werke aus dem frühen 20. Jahrhundert an dieser Stelle zunächst anachronistisch erscheinen, wurde die kuratorische Idee im Einführungstext erläutert:

Um die nationale Identität zu fördern, vereinnahmten die neuen Machthaber allegorisch aufgeladene Bildthemen verstorbener Künstler wie Hans Thoma für staats- und kulturpolitische Zwecke. Auch Friedrich Boehles muskulöse, der klassischen Antike entlehnte Männergestalten lobte die Kunstkritik des NS-Regimes als Ausdruck höchster Kampfbereitschaft und eines gesunden Volkskörpers. Der Künstler Richard Scheibe griff auf dieselben klassizistischen Vorbilder zurück und legitimierte damit sein Kunstschaffen.

Auf der linken Wand wurden die Gemälde Adam und Eva von Anton Räderscheidt (1936) und Junge Frau im Korbstuhl von Hanns Ludwig Katz (ca. 1933/34) ausgestellt (Abb. 6). Beide Künstler repräsentierten das Schaffen der als „entartet“ Verfolgten. Ihr jeweiliger Malstil und ihre Auffassungen von Körpern bildeten eine Kontrastfolie zu Scheibes idealisiertem Jüngling.

Das Konzept des Ausstellungssaals zielte mit der Auswahl der Werke darauf ab, Vorbilder, Kontinuitäten und Brüche in der Bildpolitik des Nationalsozialismus aufzuzeigen. Anders als in Nürnberg, wo es nur programmatisch aufgeladene Kunst zu sehen gibt und diese von anderen in den 1930er-Jahren existierenden Kunstkonzeptionen stark isoliert gezeigt ist, lag der Fokus im Städel Museum auf der Vereinnahmung älterer Kunst und auf der Verfolgung von Künstlern der ästhetischen

11 Einen gelungenen Überblick über die Erforschung des Themenkomplexes Kunst(politik) im Nationalsozialismus der letzten 20 Jahre bietet Zuschlag (2020).

12 Später sollte der Saal zugunsten von Sonderausstellungen thematisch anders bespielt werden.
Moderne. Dieser formalästhetische Zugang kontrastierte die zeitgleich existierenden Kunstauffassungen einerseits,

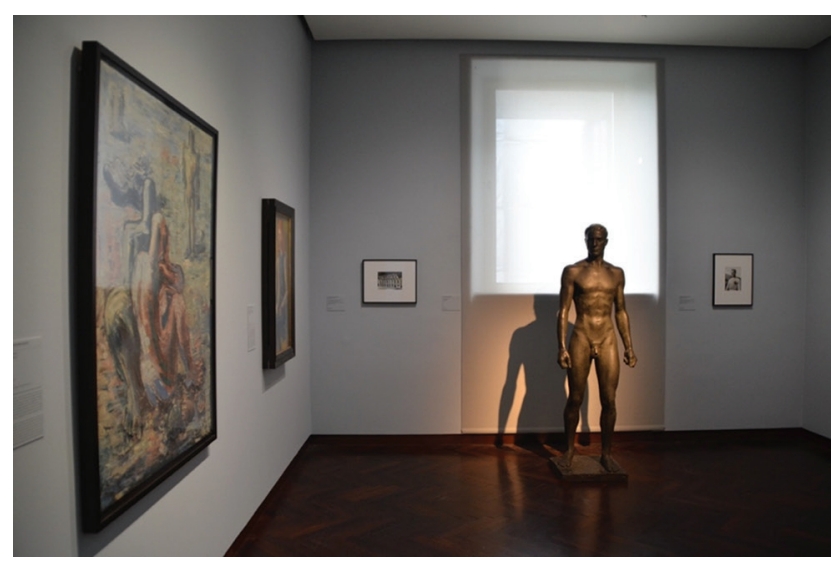

Abb. 4: Blick in den Saal „Instrumentalisierung von Kunst“ im Städe Museum, Frankfurt am Main. Linke Wand: Adam und Eva, Anton Räderscheidt (1936); Junge Frau im Korbstuhl, Hanns Ludwig Katz (circa 1933/34). Saalmitte: Zehnkämpfer, Richard Scheibe (1936).

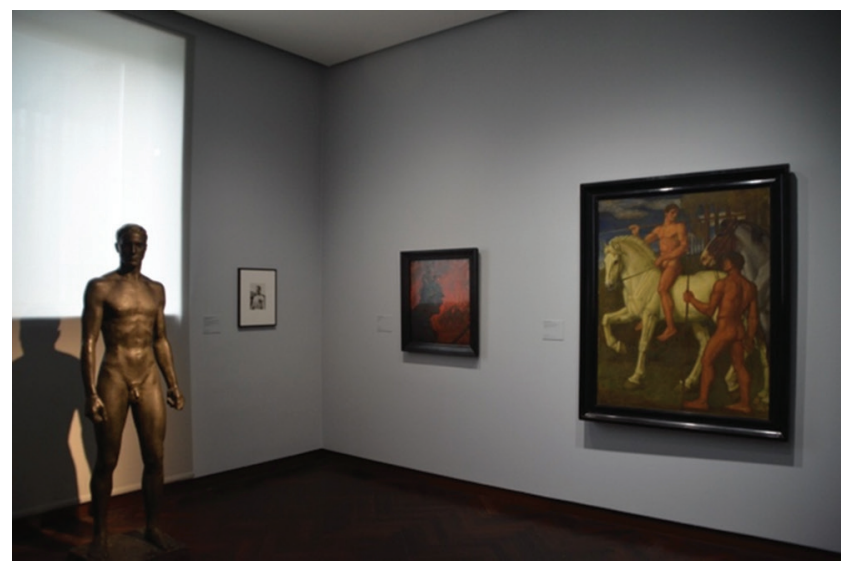

Abb. 5: Blick in den Saal „Instrumentalisierung von Kunst“ im Städel Museum, Frankfurt am Main. Rechte Wand: Der Krieg, Hans Thoma (1907); Zwei nackte Männer mit Pferden, Fritz Boehle (circa 1906).

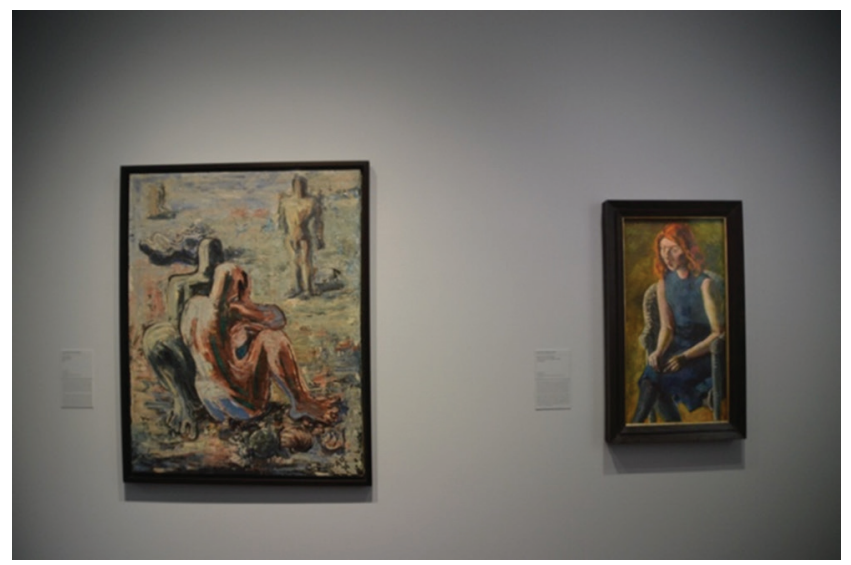

Abb. 6: Blick in den Saal „Instrumentalisierung von Kunst“ im Städel Museum, Frankfurt am Main: Adam und Eva, Anton Räderscheidt (1936); Junge Frau im Korbstuhl, Hanns Ludwig Katz (circa 1933/34). 
wie auch die klassizistischen Traditionslinien andererseits. Der Zehnkämpfer von Richard Scheibe stand dabei als einziges Exponat für die regimekonforme Kunstproduktion im Nationalsozialismus.

\section{Pinakothek der Moderne, München}

Der jüngste noch bestehende Saal zur Kunst aus dem Nationalsozialismus in der Dauerausstellung eines deutschen Kunstmuseums befindet sich in der Pinakothek der Moderne in München. Innerhalb der chronologischen Erzählung ist der Saal 13 dem Thema „Künstler im Nationalsozialismus“ gewidmet. Im Mai 2015 eröffnete das Haus zunächst die als Sonderausstellung konzipierte Präsentation "GegenKunst“, in der Werke von Otto Freundlich, Max Beckmann sowie der im Nationalsozialismus erfolgreichen Künstler Josef Thorak und Adolf Ziegler gezeigt und in ihrem kulturhistorischen Kontext diskutiert wurden (Kase 2017). Aus der Erfahrung dieser temporären Schau entstand die Konzeption der dauerhaften Präsentation zu Künstlern im Nationalsozialismus, die seit 2016 bereits zum zweiten Mal umgehängt wurde. In dem ausführlichen Raumtext, in welchem einige der im Saal präsentierten Werke kontextualisiert werden, wird das kuratorische Konzept der aktuellen Werkauswahl ersichtlich: Der Fokus liegt auf den Handlungsmöglichkeiten und Lebenswirklichkeiten der Künstler*innen.

Gleich neben dem Einführungstext hängen drei abstrakte Gemälde - K35 und Große lineare Komposition von Fritz Winter (beide 1934) sowie Ballspieler von Willy Baumeister (1935). Diese Werke versinnbildlichen die Kontinuität der abstrakten Formensprache, die - obgleich sie im Kunstbetrieb des NS-Regimes unerwünscht war - auch nach der Machtübernahme 1933 weiterhin von den Künstlern praktiziert wurde (Abb. 7). Der Präsentation im Uhrzeigersinn folgend, begegnet das Publikum an der nächsten Wand den Plastiken von Käthe Kollwitz, Herrmann Blumenthal und Gerhard Marcks. Die drei Künstler*innen verbindet, wie es im Einführungstext heißt, einerseits, dass ihre Werke

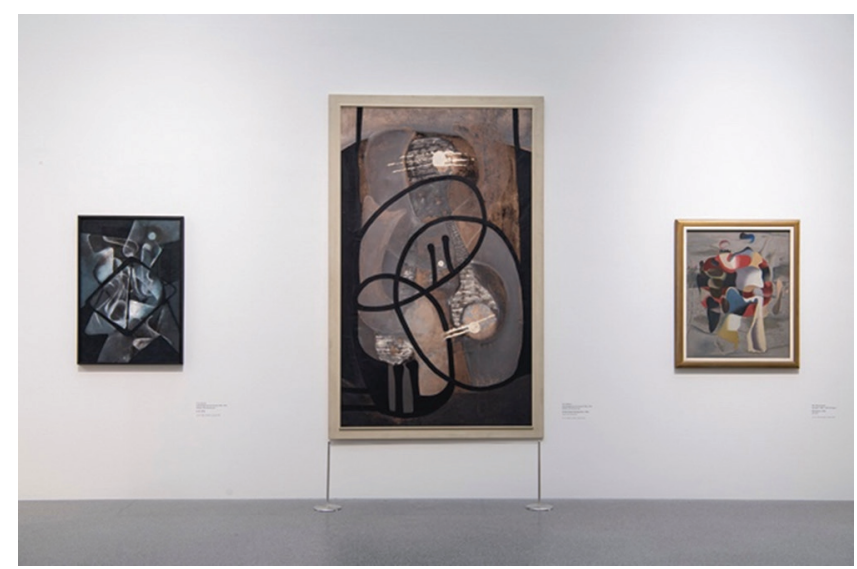

Abb. 7: Blick in den Saal „Künstler im Nationalsozialismus“ in der Pinakothek der Moderne, München, v.I.n.r.: K35 und Große lineare Komposition, Fritz Winter (beide 1934); Ballspieler, Willy Baumeister (1935). während der Beschlagnahmeaktion „Entartete Kunst“ konfisziert wurden und andererseits, dass sie „zeitweilig in der Ateliergemeinschaft Klosterstraße in Berlin verbunden [waren], in der die Sehnsucht nach einem idealen Arkadien der Realität der NS-Politik entgegentrat" (Abb. 8). Rechts neben den Plastiken hängt Karl Hofers Gemälde Mann vor Spiegel (1943).

Die Gemälde L'ange du foyer/Hausengel von Max Ernst (1937) und Der Schwager von Richard Oelze (1935) auf der nächsten Wand entstanden in Paris. Sie repräsentieren nicht nur im Exil Schaffende, sondern auch Künstler, die sich explizit mit dem Nationalsozialismus auseinandersetzten. Innerhalb des kuratorischen Konzepts dieses Saals sind sie gleichsam als „[u]nmittelbare Gegenspieler“, wie der Einführungstext verrät, zu dem wohl auffallendsten Exponat gedacht: Das großformatige Triptychon Die vier Elemente von Adolf Ziegler (1937) dominiert, umgeben von kleineren Gemälden und Plastiken, den Raum (Abb. 9-10). Wie in dem Gemälde Akt im Germanischen Nationalmuseum, beschränkte sich Ziegler auch in diesem Werk auf

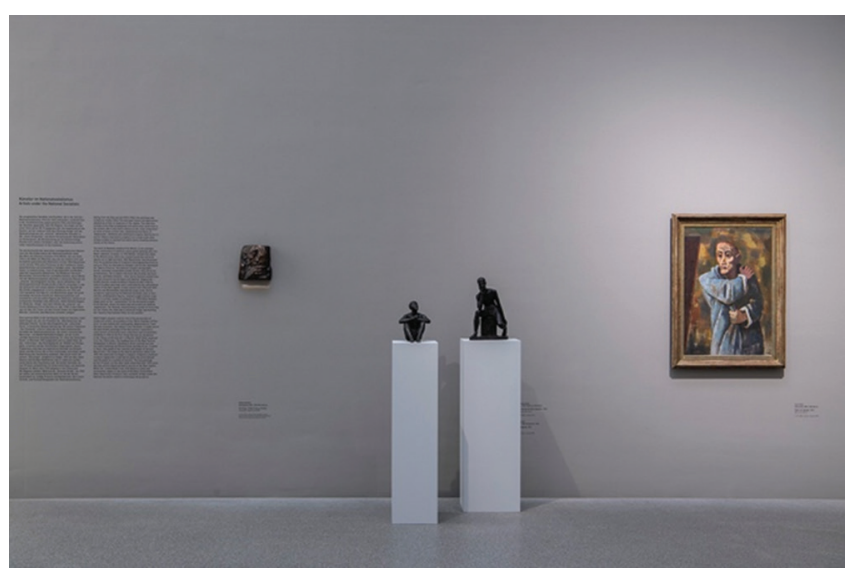

Abb. 8: Blick in den Saal „Künstler im Nationalsozialismus“ in der Pinakothek der Moderne, München, v.I.n.r.: Die Klage, Käthe Kollwitz (1938/41, Guss 1961/62); Sitzender aufschauend (Sterngucker) Herrmann Blumenthal (1936); Sitzender Jüngling, Gerhard Marcks (1937); Mann vor Spiegel, Karl Hofer (1943).

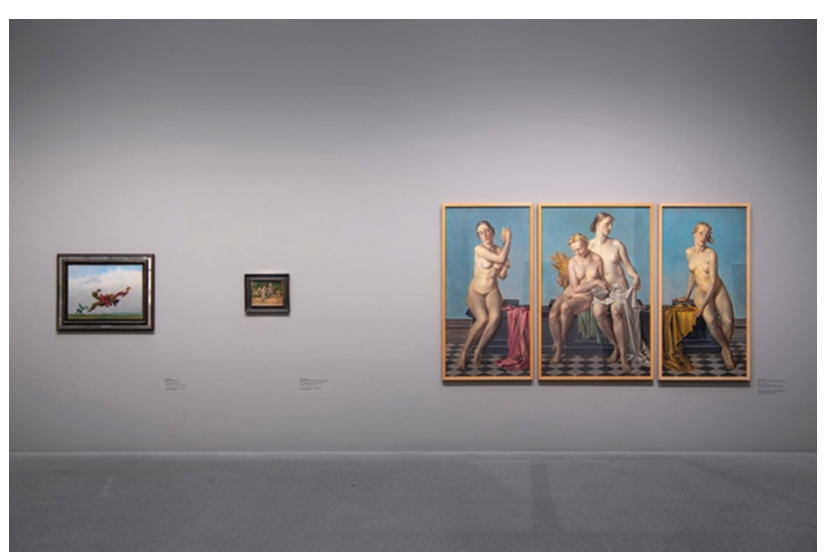

Abb. 9: Blick in den Saal „Künstler im Nationalsozialismus“ in der Pinakothek der Moderne, München, v.I.n.r.: L'ange du foyer/Hausengel, Max Ernst (1937); Der Schwager (Paar mit Hund), Richard Oelze (1935); Die vier Elemente, Adolf Ziegler (1937). Aufnahme 2018. 


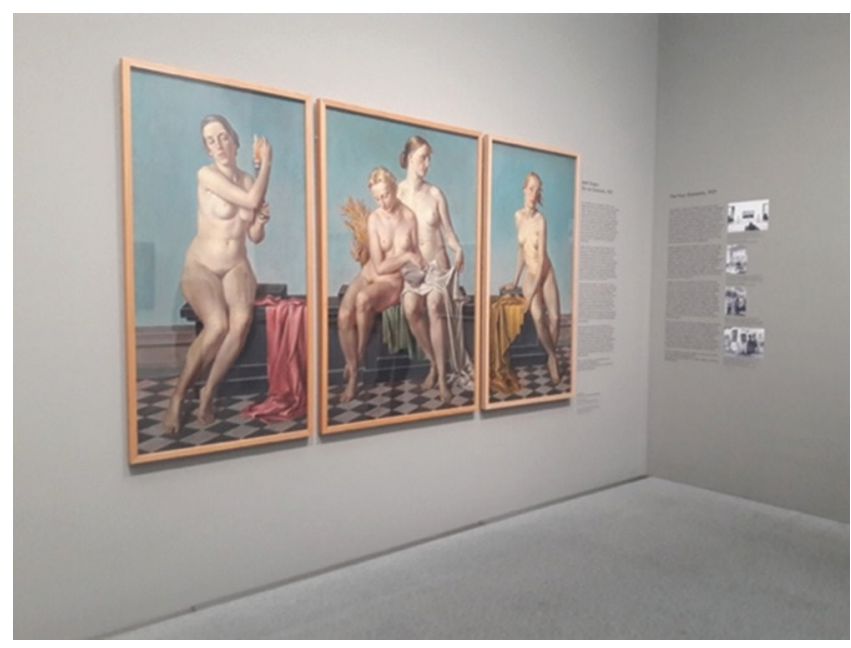

Abb. 10: Blick in den Saal „Künstler im Nationalsozialismus“ in der Pinakothek der Moderne, München: Die vier Elemente, Adolf Ziegler (1937). Aufnahme 2020 (mit ergänzter Kommentierung).

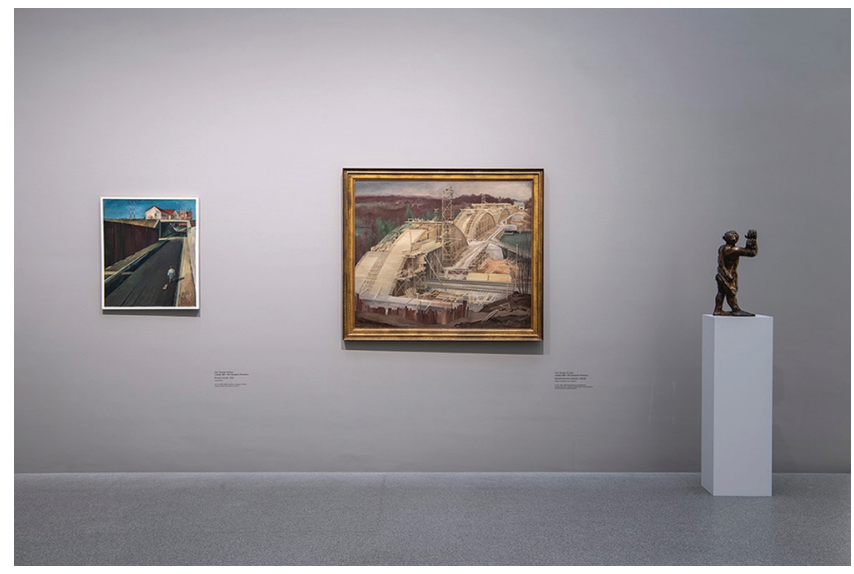

Abb. 11: Blick in den Saal „Künstler im Nationalsozialismus“ in der Pinakothek der Moderne, München: Die einsame Straße (1932) und Donaubrücke bei Leipheim (1935/36), Carl Theodor Protzen. Aufnahme 2018 (Max Beckmanns Plastik Mann im Dunkeln (1934) ist in der derzeitigen Präsentation nicht ausgestellt).

eine sitzende, weibliche Aktfigur - aber hier in vierfacher Variation.

In der allegorischen Darstellung repräsentieren die nackten, in altmeisterlicher Manier ausgeführten Frauenkörper die Elemente Feuer, Erde, Wasser und Luft (HoffmannCurtius 1989; Mittig 2014). Obwohl der Künstler insgesamt nicht einmal ein Dutzend Gemälde öffentlich ausstellte (Fuhrmeister 2015b: 65), war er einer der einflussreichsten Akteure im nationalsozialistischen Kunstbetrieb und so ist es wenig überraschend, dass seine Werke in mehreren Ausstellungen stellvertretend für die Kunst aus dem Nationalsozialismus gezeigt werden. Werk und Autor haben in diesem Fall besonders herausragende Relevanz im Hinblick auf die Kunstproduktion und -rezeption während des NS-Regimes. In seiner Funktion als Präsident der Reichskammer der bildenden Künste wurde Adolf Ziegler auch mit der Beschlagnahmeaktion „Entartete Kunst“ betraut. In diesem Saal wird er somit nicht (nur) als Maler einer altmeisterlich anmutenden - man könnte auch sagen: einer altmodischen - Aktkomposition präsentiert, sondern als ein machtvoller Akteur der nationalsozialistischen Kulturpolitik, der einen großen Einfluss darauf ausüben konnte, wer als Künstler*in tätig sein durfte und wer nicht.

Die vier Elemente sind von einem ausführlichen Objekttext begleitet. Kein anderes Werk in diesem Ausstellungssaal wird durch Objekttexte kommentiert, die Exponate sind lediglich mit knappen Objektschildern versehen, welche Künstler, Titel und Datierung benennen. Diejenigen Besucher*innen, die den langen Einführungstext nicht lesen, können die bisher besprochenen Werke ganz für sich stehend, ohne den kulturpolitischen Kontext, betrachten. Bei Adolf Zieglers Die vier Elemente ist die Kommentierung indes unübersehbar: Neben dem Triptychon ist ein langer Wandtext platziert und vier Fotoreproduktionen dokumentieren die Stationen des Gemäldes sowie die Aktivitäten Adolf Zieglers im Nationalsozialismus. Am Ende wird in dem Wandtext auf weitere Informationen auf der Website des Museums verwiesen, welche eine Broschüre zu diesem Saal, einen Aufsatz des zuständigen Kurators Oliver Kase und weitere Materialien zur Kontextualisierung umfassen. ${ }^{13}$ Nicht nur ist Die vier Elemente das einzige Werk des Saals, das diesen ausführlichen Kommentar erhält - auch auf der Website des Museums werden ausschließlich zu Saal 13 kontextualisierende Informationen bereitgestellt.

Mag die Kontrastierung der jeweils verfemten und geförderten Künstler das Narrativ bisher dominiert haben, wird es durch die letzten beiden Exponate um einige Graustufen erweitert (Abb. 11). Die beiden Gemälde Einsame Straße (1932) und Donaubrücke bei Leipheim (1935/36) von Carl Theodor Protzen sollen, dem Einführungstext zufolge, zeigen:

wie deutsche Künstler ihr Werk nach 1933 teilweise in den Dienst des NS-Regimes stellten. Während Einsame Straße von 1932 ein stilles und melancholisches Motiv im Stil der Neuen Sachlichkeit ist, huldigt Protzens Autobahnbrücke dem neuen Technik- und Fortschrittsglauben des Nationalsozialismus.

Protzens Donaubrücke bei Leipheim avancierte seit den 1970er-Jahren zu einem Sinnbild des Autobahnbildes, einer während des NS-Regimes popularisierten Bildgattung. Obwohl das Werk während des Nationalsozialismus für eine recht kurze Zeit zu sehen war - in der "Großen deutschen Kunstausstellung“ 1937 -, wurde es seit der Ausstellung „Kunst im 3. Reich“ zu dem Autobahnbild schlechthin ${ }^{14}$ und so überrascht dessen Auswahl für die Münchener Präsentation nicht. Die Gegenüberstellung mit einem weiteren Werk Protzens, aus seiner Schaffenszeit vor 1933, erlaubt hingegen einen visuellen Vergleich und dieser lässt thematische wie formalästhetische Veränderungen auf Anhieb erkennen. An dieser Stelle verschwimmt

13 https://www.pinakothek.de/gegenkunst (Zugriff am 30. November 2021). Die in der Broschüre auf der Webseite besprochenen Werke entsprechen der ersten Hängung des Saals.

14 So die Kunsthistorikerin Anke Gröner auf der Tagung „Kunst im Nationalsozialismus. Forschungsfragen, Forschungsperspektiven, Forschungsinfrastrukturen" am 12. Oktober 2021 am Münchner Zentralinstitut für Kunstgeschichte. Ihr Vortrag „Zu Gemälden der Reichsautobahn von Carl Theodor Protzen“ resümierte die Forschungsergebnisse ihrer noch unveröffentlichten Dissertationsschrift zur Carl Theodor Protzens @Euvre. 
die Dichotomie „Künstler der Moderne“ versus „Künstler des Nationalsozialismus“. In der Präsentation dieser beiden Bilder werden unterschiedliche Kunstauffassungen eines Malers evident, der sich mit den Bilderwelten des neuen Regimes aktiv auseinandersetzte und seinen Platz in dem neuen kulturpolitischen System suchte. Ein weiterer Vergleich mit dem Saal des Germanischen Nationalmuseums lässt den veränderten Umgang mit der Kunst aus dem Nationalso-zialismus seit den späten 1990er-Jahren erkennen: Nicht die eindeutige Zuordnung eines Künstlers oder Werks zur Kategorie „NS-Kunst“ steht in München im Vordergrund, sondern die Diskussion individueller Kunstauffassungen und Handlungsfelder.

\section{KANON UND KONFLIKT}

Gibt es heute, im Jahr 2022, überhaupt einen Konflikt, wenn es um die Präsentation NS-konformer Kunst in den Kunstmuseen geht? Mit Blick auf die teils polemischen und tabuisierenden Debatten der 1970er- bis 1990er-Jahre, die den Diskurs über diese Kunst und ihre öffentliche Zurschaustellung dominierten, lässt sich im 21. Jahrhundert eine auf viele Ursachen zurückzuführende Ausdifferenzierung beobachten. Eine Selbstverständlichkeit ist die regimekonforme Kunst aus dem Nationalsozialismus in einem Kunstmuseum aber deshalb noch lange nicht. Das zeigt nicht zuletzt das umfassendere Vermittlungsangebot: Sowohl im Germanischen Nationalmuseum als auch in der Pinakothek der Moderne wurde für die Präsentation der Kunst aus dem Nationalsozialismus auf eingehende Kommentierung zurückgegriffen - durch eine künstlerische Arbeit in Nürnberg und durch zusätzliche Text- und Bildmaterialien in München. Begreift man Ausstellungen als Vermittlungsmedien, welche durch die Auswahl der Künstler*innen sowie die Anordnung ihrer Werke im Raum „Wissensordnungen konstituieren“ (Hoffmann 2013: 135), wird die Diskrepanz in der Präsentation der Kunst aus dem Nationalsozialismus und derjenigen der ästhetischen Moderne evident. Da die nationalsozialistische Kulturpolitik und die künstlerischen Lebenswirklichkeiten zwischen 1933 und 1945 bislang nicht oder nur selten zum „Repertoire“ von Kunstmuseen zählten, müssen die in den Ausstellungen gezeigten Werke, Akteure und Kontexte einer breiten Öffentlichkeit überhaupt erst vorgestellt werden.

Ausstellungen sind aber nicht nur Räume der Wissensproduktion, sondern in innen spiegeln sich der Wissensstand und der Kunstbegriff der jeweiligen Zeit wider. Die „Kunst im Nationalsozialismus“, wie der Saal im Germanischen Nationalmuseum betitelt ist, auf die Ankäufe Adolf Hitlers auf den "Großen Deutschen Kunstausstellungen" zu beschränken, ist aus heutiger Sicht zwar verkürzt, doch gilt es zu bedenken, dass zum Zeitpunkt der Fertigstellung des Ausstellungsdisplays kaum Grundlagenforschung zu diesem Themenkomplex vorlag. Keine einzige Monografie widmete sich ausschließlich den
"Großen Deutschen Kunstausstellungen“, während sie in so gut wie jedem Überblickswerk zum Thema NS-Kulturpolitik wie selbstverständlich erwähnt wurden. Zudem galt in der Kunstgeschichte lange - und dies nicht zuletzt in Folge der Ausstellung „Kunst im 3. Reich“ und der zeitgleich erschienenen Monografie von Berthold Hinz (Hinz 1974) - diejenige Kunst, welche die NS-Publizistik stark popularisierte, als die NS-Kunstproduktion schlechthin (Fuhrmeister 2013: 194).

Im Städel Museum wurde ein anderer Erzählmodus gewählt. Richard Scheibe stand gleichsam für die Vereinnahmung der neoklassizistischen Vorbilder im Nationalsozialismus und als Gegenpol zu den verfemten Künstlern. Die Komplexität seiner künstlerischen Biografie wurde indes nicht ausgeführt: Scheibe war vor, während und nach dem Nationalsozialismus als Künstler und Kunstprofessor erfolgreich. 1925 erhielt der Bildhauer einen Ruf an das Städelsche Kunstinstitut (die Kunsthochschule in Frankfurt am Main) und war dort als Professor tätig. Nach zunächst einer Entlassung im März 1933, sollte er bereits im Oktober des Jahres wieder in sein Amt eingesetzt werden und wechselte 1935 nach Berlin an die Preußische Akademie der Künste. Während des NS-Regimes wurde Scheibe mit Aufträgen bedacht, stellte kontinuierlich auf den "Großen Deutschen Kunstausstellungen" aus und wurde in die Liste der "Gottbegnadeten“ Künstler aufgenommen, die vom Kriegseinsatz befreit bleiben sollten (Eckstein 2005: 323f.; Brauneis/Gross 2021: 93). Nach 1945 erhielt Scheibe eine Professur an der Berliner Hochschule für bildende Künste und wurde durch Ämter und Ehrungen (u.a. 1953 mit dem Bundesverdienstkreuz) ausgezeichnet. Die Kontinuität seines Schaffens ist beispielhaft für eine Reihe von Künstlern, die insbesondere im Bereich der Bauplastik tätig waren (Brauneis 2020). Scheibes vielschichtige Künstlerbiografie hätte dem Frankfurter Ausstellungskonzept ermöglicht, weitere Traditionslinien aufzugreifen: im Hinblick auf die klassizistische Formensprache, die Scheibes OEuvre über die Grenzen politischer Systeme hinaus aufweist, aber auch mit Blick auf die personellen Kontinuitäten der Kunstproduzent*innen.

Der Kurator des besprochenen Ausstellungssaals in der Pinakothek der Moderne, Oliver Kase, schreibt in einem Aufsatz: „An der Frage des Umgangs mit NS-Kunst wird das Kunstmuseum mehr denn je belegen müssen, ob es sich nur mehr als ästhetisches Institut der Geschmacksbildung und der Präsentation eines exklusiven Kanons der Moderne versteht oder auch als demokratische Einrichtung des kritischen gesellschaftlichen Diskurses“ (Kase 2017: 323). Die Auffächerung der unterschiedlichen Lebenswirklichkeiten der Künstler*innen ist eine wichtige Grundlage für diesen Diskurs. Dies gilt sowohl für die Künstler*innen, die während des NS-Regimes erfolgreich waren, als auch für diejenigen, die zum etablierten Kanon der Moderne zählen. Mit Blick auf die Werkauswahl in der Pinakothek der Moderne, ist mit Carl Theodor Protzen ein Künstler vertreten, der in Sujet wie Darstellungsweise den Erwartungshaltungen des neuen 
Regimes nachkam und dadurch im offiziellen Kunstbestrieb vertreten war. Solche bewussten Selbstverortungen und stilistischen Neujustierungen eines Künstlers innerhalb der nationalsozialistischen Kulturpolitik sind eine Facette, anhand derer die Komplexität des künstlerischen Schaffens während des NS-Regimes greifbar wird.

Auch am Beispiel der beiden im selben Saal präsentierten Bildhauer Hermann Blumenthal und Gerhard Marcks ließen sich solche Grauschattierungen diskutieren. Die ambivalenten Biografien dieser Künstler enthalten sowohl Momente der Verachtung wie der Beachtung ihres Schaffens in der NS-Zeit. Hermann Blumenthal hatte seinen vorläufigen Höhepunkt noch vor der Machtübernahme erlebt: 1930, mit 25 Jahren, erhielt er den renommierten Großen Staatspreis der Preußischen Akademie der Künste, der inm einen Aufenthalt in der Villa Massimo in Rom ermöglichte. Nach 1933 änderte sich die Situation des aufstrebenden Künstlers. Er wurde nicht mehr zu Akademie-Ausstellungen zugelassen, seine Bronzeplastik Schreitender wurde 1937 aus dem Folkwang-Museum in Essen als „entartet" beschlagnahmt und vier weitere Arbeiten sollten in der Berliner Galerie Buchholz nicht ausgestellt werden dürfen (Berger 1994: 75; Scholz 2015: 199-203). Ungeachtet dessen erhielt Blumenthal während des NS-Regimes jedoch auch Anerkennung: Ein weiteres Stipendium für einen Aufenthalt in der Villa Massimo wurde inm 1936/37 zuerkannt und 1939 verlieh die Stadt Düsseldorf dem Bildhauer den Cornelius-Preis, der seit 1936 zur „Förderung der ,zeitgemäßen ' Kunst" vergeben wurde. Mit einer Arbeit beteiligte er sich an den „Großen deutschen Kunstausstellungen“ - 1941 war dort sein Porträtkopf der Schauspielerin Ruth Gerntholtz zu sehen. ${ }^{15}$

Gerhard Marcks, der in der Pinakothek der Moderne wie Hermann Blumenthal - stellvertretend für die Berliner Künstlergemeinschaft Klosterstraße und deren „Sehnsucht nach einem idealen Arkadien“ steht, musste ebenfalls erleben, wie seine Werke 1937 als „entartet“ diffamiert wurden. Nachdem er mit der Machtübernahme als Leiter der Bildhauer-Werkstatt an der Burg Giebichenstein in Halle entlassen worden war, bedeutete die Verfemung seiner Werke einen weiteren immensen Einschnitt für den Bildhauer. Doch bemühte er sich während des Regimes fortwährend um öffentliche Aufträge: Er bewarb sich unter anderem um die Mitwirkung an der bauplastischen Ausstattung des Berliner Reichssportfelds und gestaltete, wie er selbst schrieb, „Porzellanvasen für die Reichskanzlei“ (Bushart 1993: 110). Wie die Kunsthistorikerin Magdalena Bushart bereits 1993 darlegte, war Marcks' künstlerischer Werdegang während des Nationalsozialismus ein „Taktieren zwischen Verweigerung und der Suche nach Anerkennung" (ebd.). Zwar wurden dem einstigen Leiter der Bauhaus-Töpferei die meisten Aufträge während des Nationalsozialismus verwehrt (auch die Vasen wurden ihm 1938 entzogen) und die Lehrtätigkeit als Nachfolger von seinem Freund Richard

15 Siehe den Datensatz zu Hermann Blumenthals Bronzekopf „Porträt Ruth Gernholtz in der Online-Datenbank GDK-Research: http://www.gdk-research.de/de/obj19363648.html (Zugriff am 30. November 2021).
Scheibe erhielt er an der Städelschule aufgrund seiner politischen Bewertung nicht, doch noch 1936 schrieb er von "guten Grundideen des Nationalsozialismus" (Bushart 1993: 105). Diese kursorisch beschriebenen Lebensstationen von Hermann Blumenthal und Gerhard Marcks erscheinen widersprüchlich und doch sind sie Teil ihrer künstlerischen Biografien.

Mit Blick auf Künstler wie Carl Theodor Protzen, Richard Scheibe, Hermann Blumenthal und Gerhard Marcks stelle ich außerdem fest, dass der Konflikt in der musealen Präsentation von Kunst aus dem Nationalsozialismus nicht nur darin besteht, dass das Gros der im NS-Regime prominenteren Akteure einer breiten Öffentlichkeit kaum bekannt ist und einer intensiveren Vermittlung bedarf. Auch den Biografien von Künstlern der ästhetischen Moderne während des Nationalsozialismus wird weniger Aufmerksamkeit zuteil. Wie positionierten sich die Künstler*innen der Moderne zum neuen politischen System und dessen Ideologie? An welchen offiziellen Wettbewerben nahmen sie teil, wo stellten sie aus? Die eingangs erwähnten Ausstellungen zu Emil Nolde und den Künstlern der Brücke zeigten eindrücklich, wie sich einige der Maler innerhalb des neuen politischen Rahmens einrichteten.

Die Chronologie der drei vorgestellten Ausstellungsräume ist zugleich ein Spiegel der Forschungstendenzen. Bezieht man auch die erwähnten Sonderausstellungen in die Betrachtung mit ein, zeigt sich, dass die kuratorischen Konzepte sich von dem Ziel, die vermeintlich typische „NS-Kunst“ aufzuzeigen, in den vergangenen Jahren zugunsten der Ambivalenzen in der NS-Kunstpolitik verschoben haben. Am Beispiel Emil Nolde konnte auch einer breiten Öffentlichkeit verdeutlicht werden, wie komplex dieses Themenfeld ist und wie ungenügend die Kategorien „NS-Kunst“, „Unkunst“, „Propaganda“ oder „Kitsch“ den Gegenstand erfassen, wenn es um die Integrität der Künstler*innen ging. Daran offenbart sich erst die Notwendigkeit, Werk und Biografie der Künstler*innen in ihrer Gesamtheit in den Blick zu nehmen, um die (Un)Möglichkeiten ihres künstlerischen Daseins während des Nationalsozialismus mit all den Paradoxien zu erfassen. Solange jedoch nur die Museumsräume, welche explizit die Kunst aus dem Nationalsozialismus thematisieren, sich solchen Grauschattierungen und Paradoxien widmen, bleibt der Konflikt mit dem Kanon bestehen: Hier die Qualität, die Meisterwerke und der ästhetische Genuss - dort die „schwierigen“ Werke und die kulturhistorische Kontextualisierung.

Welches Narrativ in einer Ausstellung präsentiert werden kann, hängt in erster Linie aber natürlich auch von den vorhandenen Sammlungsbeständen ab. So entstammen die beiden Werke Die vier Elemente von Adolf Ziegler und Donaubrücke bei Leipheim von Carl Theodor Protzen beispielsweise dem Kunstkonvolut „Überweisungen aus dem Staatsbesitz“, das die Bayerischen Staatsgemäldesammlungen verwahren. Die Gemälde im Germanischen Nationalmuseum stammen aus der Sammlung „Ehemaliger Reichsbesitz“ und können als Leihgaben des Deutschen Historischen Museums, das 
diesen Bestand dauerhaft aufbewahrt, gezeigt werden. Beide Konvolute gehen auf Sammlungen von NS-Funktionären sowie NS-Institutionen zurück und wurden in der Nachkriegszeit in museale Bestände überführt. Doch auch bereits während des NS-Regimes gelangte zeitgenössische Kunst in die Sammlungen deutscher Kunstmuseen. Ein genauer Blick auf diesen Aspekt der institutionellen Sammlungsgeschichte innerhalb der Ausstellungsdisplays könnte die Vermittlung der nationalsozialistischen Kulturpolitik um eine Facette erweitern.

\section{Abbildungsnachweise}

Abb. 1-3, 10: Foto: Darja Jesse, mit freundlicher Genehmigung des Germanischen Nationalmuseums, Nürnberg, und der Pinakothek der Moderne, München.

Abb. 4-6: () Städel Museum, Frankfurt am Main.

Abb. 7-9, 11: Pinakothek der Moderne „Künstler im Nationalsozialismus“. Seit 2018. Foto: Haydar Koyupinar, Bayerische Staatsgemäldesammlungen.

Für alle abgebildeten Kunstwerke wurden die Urheberrechte bei VG Bild-Kunst, Bonn, eingeholt.

\section{Literatur}

Berger, Ursel (1994²), „,Auf dem Wege nach Griechenland“ - Die Bildhauer Hermann Blumenthal und Ludwig Kasper", Akademie der Künste Berlin (Hg.), Ateliergemeinschaft Klosterstraße Berlin 1933-1945. Künstler in der Zeit des Nationalsozialismus, Berlin, Edition Hentrich, 70-77.

Berswordt-Wallrabe, Silke von (2016), „Artige Kunst‘ - zur Einführung“, Dies. / Neumann Jörg-Uwe / Tieze Agnes (Hg.), Artige Kunst. Kunst und Politik im Nationalsozialismus, Bielefeld, Kerber art, 10-15.

Brauneis, Wolfgang (2020), „,Gottbegnadete ' in der Bundesrepublik. Künstler des Nationalismus in der Nachkriegsmoderne", Historische Urteilskraft. Magazin des Deutschen Historischen Museums, 2, 41-44.

Brauneis, Wolfgang / Gross, Raphael (Hg.) (2021), Die Liste der "Gottbegnadeten“. Künstler des Nationalsozialismus in der Bundesrepublik, München, Prestel Verlag.

Bushart, Magdalena (1993), „Ein Bildhauer zwischen zwei Stühlen. Gerhard Marcks in den dreißiger Jahren", Nerdinger Winfried (Hg.), Bauhaus-Moderne im Nationalsozialismus. Zwischen Anbiederung und Verfolgung, München, Prestel, 103-112.

Bussmann, Georg $\left(1975^{4}\right)$, „Zu dieser Ausstellung“, Frankfurter Kunstverein (Hg.), Kunst im 3. Reich. Dokumente der Unterwerfung, Frankfurt am Main, Zweitausendeins Verlag, 3.

Deutscher Museumsbund e.V. (gemeinsam mit ICOM-Deutschland) (2006), Standards für Museen, Kassel, Berlin. URL: https://www. museumsbund.de/wp-content/uploads/2017/03/standards-fuermuseen-2006-1.pdf

Eckstein, Beate (2005), Im öffentlichen Auftrag. Architektur- und Denkmalplastik der 1920er bis 1950er Jahre im Werk von Karl Albiker, Richard Scheibe und Josef Wackerle, Hamburg, Kovač.

Frankfurter Kunstverein (ca. 1975), Betrifft: Reaktionen. Anlaß: Kunst im 3. Reich. Dokumente der Unterwerfung. Ort: Frankfurt, Frankfurt am Main, Frankfurter Kunstverein.

Fuhrmeister, Christian (2013), „Die ,Große Deutsche Kunstausstellung' 1938. Relektüre und Neubewertung“, Atlan Eva / Crüwell Konstanze / Voss Julia (Hg.), 1938. Kunst, Künstler, Politik, Göttingen, Wallstein, 190-207.

Fuhrmeister, Christian (2015a), „Die Große Deutsche Kunstausstellung 1937-1944“, Ruppert Wolfgang (Hg.), Künstler im Nationalsozialismus. Die „Deutsche Kunst“, die Kunstpolitik und die Berliner Kunsthochschule, Köln / Weimar / Wien, Böhlau, 97-106.
Fuhrmeister, Christian (2015b), „Adolf Ziegler - Maler, Präsident der Reichskammer der bildenden Künste, Maler“, Benz Wolfgang / Eckel Peter / Nachama Andreas (Hg.), Kunst im NS-Staat. Ideologie, Ästhetik, Protagonisten, Berlin, Metropol, 59-72.

Habsburg-Lothringen, Bettina (2012), „Dauerausstellungen. Erbe und Alltag“, Dies. (Hg.), Dauerausstellungen. Schlaglichter auf ein Format, Bielefeld, transcript, 9-18.

Hinz, Berthold (1974), Die Malerei im deutschen Faschismus. Kunst und Konterrevolution, München, Hanser.

Hoffmann, Katja (2013), Ausstellungen als Wissensordnungen. Zur Transformation des Kunstbegriffs auf der Documenta 11, Bielefeld, transcript.

Hoffmann, Meike / Scholz, Dieter (Hg.) (2020), Unbewältigt? Ästhetische Moderne und Nationalsozialismus. Kunst, Kunsthandel, Ausstellungspraxis, Berlin, Verbrecher Verlag.

Hoffmann-Curtius, Kathrin (1989), „Die Frau in ihrem Element. Adolf Zieglers Triptychon der ,Naturgesetzlichkeit', Kritische Berichte, 2, 5-30.

Imdahl, Georg (1988), „Pose und Indoktrination. Zu Werken der Plastik und Malerei im Dritten Reich", Staeck Klaus (Hg.), Nazi-Kunst ins Museum?, Göttingen, Steidl, 87-99.

Kase, Oliver (2017), „Die Ausstellung GegenKunst in der Pinakothek der Moderne. Konzept - Reaktionen - Konsequenzen“, Fuhrmeister Christian / Hauser-Mair Monika / Steffan Felix (Hg.), Vermacht. Verfallen. Verdrängt. Kunst und Nationalsozialismus. Die Sammlung der Städtischen Galerie Rosenheim in der Zeit des Nationalsozialismus und in den Nachkriegsjahren, Petersberg, Michael Imhof, 322-335.

Langfeld, Gregor (2018), „The canon in art history. Concepts and approaches", Journal of Art Historiography, 19, o.S. [GL1]. URL: https://arthistoriography.files.wordpress.com/2018/11/langfeld.pdf Locher, Hubert (2013), „Kunstwissenschaft", Rippl Gabriele / Winko Simone (Hg.), Handbuch Kanon und Wertung. Theorien, Instanzen, Geschichte, Stuttgart, J.B. Metzler, 364-371.

Mittig, Hans-Ernst (2014), „Offene Kapitel beim Umgang mit NS-Kunst in Museum, Ausstellung und Forschung", RIHA Journal 0098 (09 October 2014), o.S. DOI: https://doi.org/10.11588/ riha.2014.0.69956

Muttenthaler, Roswitha / Wonisch, Regina (2006), Gesten des Zeigens. Zur Repräsentation von Gender und Race in Ausstellungen, Bielefeld, transcript. 
Peters, Ursula (2001), „Über die Schnittstelle zwischen Vollkommenheitswunsch und Unmenschlichkeit. ,Walter at the Helm of Beauty von Jochen Gerz", Monatsanzeiger. Museen und Ausstellungen in Nürnberg, 245, $8 f$.

Schmidt, Marlies (2012), Die „Große Deutsche Kunstausstellung 1937 im Haus der Deutschen Kunst zu München“. Rekonstruktion und Analyse, Halle (Saale). DOI: http://dx.doi. org/10.25673/823

Scholz, Dieter (2015), „Hermann Blumenthal, Fräulein Niemöller, 1935“, Ders. I Obenaus Maria (Hg.), Die schwarzen Jahre. Geschichten einer Sammlung 1933-1945, Berlin, Verbrecher Verlag, 199-203.

Soika, Aya / Fulda, Bernhard / Ring, Christian (2019), Emil Nolde. Eine deutsche Legende. Der Künstler im Nationalsozialismus, 2 Bde., München / London / New York, Prestel.
Soika, Aya / Hoffmann, Meike (2019), „Einleitung“, Dies. (Hg.), Flucht in die Bilder? Die Künstler der Brücke im Nationalsozialismus, München, Hirmer, 13-16.

Staeck, Klaus (1988), Nazi-Kunst ins Museum?, Göttingen, Steidl.

Weishaupt, Sonja (2005), „Nazikunst ins Museum? Zum Umgang mit NS-Künstlern in deutschen Ausstellungen“, Blask Falk / Friedrich Thomas (Hg.), Menschenbild und Volksgesicht. Positionen zur Porträtfotografie im Nationalsozialismus. Berliner Blätter. Ethnographische und ethnologische Beiträge, Sonderheft 36, 32-37.

Zuschlag, Christoph (2020), „Kunst und Kunstpolitik im Nationalsozialismus. Eine Forschungsbilanz der letzten 20 Jahre“, Hoffmann Meike / Scholz Dieter (Hg.), Unbewältigt? Ästhetische Moderne und Nationalsozialismus. Kunst, Kunsthandel, Ausstellungspraxis, Berlin, Verbrecher Verlag, 14-35. 